\title{
A Successful Collaboration Between an Urban School District, a Health System, and a Public Health Department to Address COVID-19 While Returning Children to the Classroom
}

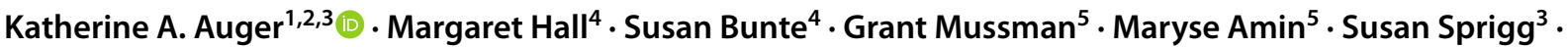 \\ Lauren Porter ${ }^{3} \cdot$ Rita Porter $^{5} \cdot$ Mona Mansour ${ }^{2,6} \cdot$ Mary Carol Burkhardt $^{2,6} \cdot$ Robert S. Kahn $^{2,3,6}$
}

Accepted: 12 January 2022 / Published online: 26 February 2022

(c) The Author(s), under exclusive licence to Springer Science+Business Media, LLC, part of Springer Nature 2022

\begin{abstract}
We sought to create and implement a set of COVID-19 mitigation processes including reliable testing to minimize in-school transmission of SARS-CoV-2. A large urban school district (>33,000 students), a city health department, and a free-standing children's hospital partnered to implement multi-layered mitigation procedures which included access to polymerase chain reaction (PCR) testing with same day or next morning results. We tracked COVID-19 cases as well as probable/confirmed transmissions and identified needed mitigations through frequent huddles. During the 2020-2021 school year, there were 13 weeks of hybrid in person learning and 9 weeks of 5 day a week learning. Of the 1936 cases documented, only $3.2 \%$ resulted in subsequent school-related transmission. When children felt ill in the classroom, they were isolated within $10 \mathrm{~min}$ of reporting ill symptoms ( $>90 \%$ of the time). PCR test results were routinely available to the school district by $6 \mathrm{AM}$ the following morning (79-99\% of the time, depending on the learning model). An adaptive, fast-learning partnership across school district, public health, and a children's hospital minimized school-related transmission of COVID-19 and allowed children to safely return to the classroom.
\end{abstract}

Keywords COVID-19 $\cdot$ School $\cdot$ Health systems $\cdot$ Public health

Katherine A. Auger

katherine.auger@cchmc.org

1 Division of Hospital Medicine, Cincinnati Children's Hospital Medical Center, 3333 Burnet Ave, Cincinnati, OH 45229, US

2 Department of Pediatrics, University of Cincinnati College of Medicine, 2600 Clifton Ave, Cincinnati, OH 45221, US

3 James M. Anderson Center for Health Systems Excellence, 3333 Burnet Ave, Cincinnati, OH 45229, US

4 Cincinnati Public School System, 2651 Burnet Ave, Cincinnati, OH 45219, US

5 Cincinnati Health Department, 3101 Burnet Ave, Cincinnati, OH 45229, US

6 Division of General and Community Pediatrics, 3333 Burnet Ave, Cincinnati, OH 45229, US

\section{Background}

COVID-19 has been profoundly disruptive to children. Not only have more than 12 million children contracted the disease [1], children who have not contracted COVID-19 have seen their daily lives greatly disrupted. One of the biggest sources of this disruption has been the dramatic changes to schools and school environments during the pandemic. School systems have been forced to adapt to unprecedented circumstances without evidence to guide their decisionsbalancing risk from a lethal infectious disease [2, 3], with their mission to educate children and foster childhood social and emotional development. Dramatic shifts in teaching modes-including closures, hybrid schedules, and remote teaching - have significant implications for overall educational attainment, as well as the potential to widen existing educational equity gaps [4].

Returning students to classrooms as safely as possible involves a high level of complexity and tremendous number of unknowns. While guidelines for school policies continue to emerge and evolve [5, 6], there is a paucity of specifics, 
and no roadmap for processes that must bridge schools, public health, and health care. Thus, it is critical that school systems adapt and partner with health and public health experts with a goal of providing safe, in-person school options.

As the Cincinnati public school (CPS) district began contemplating strategies to optimize learning in the 2020-21 school year, the district capitalized on existing partnerships with Cincinnati children's hospital medical center (CCHMC) and the Cincinnati health department (CHD) to design, implement, and test COVID-19 testing and mitigation strategies. The goals of this partnership were to work through the complexity and uncertainty by sharing a learning system approach, and to work across sectors to gain clear vision, aim, drivers, and critical process measures. CPS students returned to the classroom in October 2020 in a hybrid model, with two cohorts of children each with in-person learning occurring 2 days a week. There was a subsequent all-remote period during the winter months (November 23-January 31) followed by another hybrid in-person learning period February 1-March 19. Full in-person instruction began after spring break on March 31, 2021 through the end of the school year in late May. In this report, we present our experiences, challenges, learnings, and successes.

\section{Methods}

\section{Setting}

CPS district is a large, urban district with a high percentage of economically disadvantaged families; approximately $80 \%$ of children in the district qualify for free or reduced-price lunch. CPS consists of 66 schools and serves 33,290 children living in southwest Ohio.

\section{Goal}

Our goal was to minimize in-school transmission. We sought to achieve this goal by developing a system to quickly identify, isolate, and remove any students with COVID-19 from the school building. This required offering reliable and easy access to COVID-19 PCR testing in each school building for symptomatic students, with rapid isolation of the ill student, next-morning access to COVID-19 test results, and expeditious identification of potential close contacts to begin quarantine. Schools also implemented a robust bundle of in-class protective measures in addition to in-school COVID testing, including masking, and distancing.

\section{Stakeholder Team}

To address the challenges with school reopening during the pandemic, we assembled a multi-stakeholder team (Online Fig. 1). This team included leaders from CPS; CHD school nurse leaders, clinicians, and epidemiologists; CCHMC quality improvement (QI) teams, population health leaders, and clinical laboratory leaders; and representation from school-based health centers. CPS has longstanding partnerships with both CCHMC and CHD. CPS and CCHMC have worked together around QI initiatives and training; CHD provides school health services to CPS through the support of school nurses and schoolbased health centers.

\section{Collaborative Design and Improvement Sessions}

To achieve this goal, we convened a series of in-depth design sessions. The initial design meetings were in June 2020 with a wide range of stakeholders. The three partner organizations CPS, CCHMC, and CHD gathered with approximately 50 community representatives, including students and parents. Team members gathered to discuss how to implement COVID-19 mitigation for the upcoming school year. Topics discussed included:

- Whether and how to screen for COVID-19 symptoms among students and staff

- Responses when a child screened positive

- COVID-19 testing opportunities in collaboration with CCHMC and CHD

After the design sessions the team conducted regular meetings between the three institutional partners over video link. CCHMC QI specialists provided support to map in detail the critical processes from beginning to end of the testing and quarantine effort before school began (Online Fig. 2).

\section{COVID-19 Testing Process}

When a student had possible COVID-19 symptoms upon arrival to school or during the school day, the child was immediately sent to a COVID-19 isolation room and the school nurse was notified. All CPS staff were trained in screening and identification of COVID-19 symptoms. The CHD school nurse performed a health assessment in the isolation room. If the child's symptoms were consistent with SARS-CoV-2 infection, a COVID-19 test was offered, and collected by the nurse. Parents and guardians signed standing consent forms in the case that a student became 
symptomatic during the year, these consent forms also gave permission for test results to be released to the school. If a standing consent form was not on file, the guardian was offered the option of signing the form during pickup of the ill child. Students with potential COVID-19 symptoms who declined testing were sent home to quarantine with a letter recommending testing with locations.

All testing swabs were collected by courier twice daily and taken to CCHMC for testing. All tests were polymerase chain reaction (PCR) tests to offer the highest sensitivity and specificity. CCHMC runs PCR testing multiple times a day allowing test results to often be available the same day. Positive results were communicated to CHD as the public health authority and to a CPS hotline. To ensure redundancy in this system, positive results were also sent to school nurses each morning via secured email, with a goal that the test result be known by 6AM the next day for appropriate isolation of close contacts. School nurses contacted families with the results. Upon being notified of a positive case, the CPS principal, teachers, and CHD nurse worked together to identify close contacts, who were notified and asked to quarantine.

For children with medical insurance, the insurance was billed for the testing, the family was not charged for any out-of-pocket costs. For children without insurance, testing costs were covered by federal funds via the state of Ohio to CCHMC for implementation of a robust community testing infrastructure.

\section{Iteration of Procedures and Refinement of Processes}

Once in-person learning began, a daily huddle was conducted before school each morning. The huddle was an opportunity to discuss cases, escalate issues or challenges, and share learnings as testing processes evolved. This huddle included operational leaders from CPS, CHD, and CCHMC.

\section{Measures}

The multidisciplinary team discussed potential measures of success, including two outcome and four process measures that would be meaningful for school processes and COVID19 disease incidence. We focused on two outcome measures: (1) overall number of COVID-19 cases in both students and teachers/staff and (2) number of suspected/confirmed inschool transmissions. Key process measures included: (1) time from a child feeling unwell (as recorded by the student's teacher or recorded at the time of the positive daily screening) to isolation in the designated isolation room at school, (2) number of students who entered the isolation room each day, (3) number of COVID-19 tests collected daily and (4) percentage of COVID-19 test results available before the start of the next school day.

\section{Key Protective Measures}

The bundle of protective measures implemented by all schools included: universal masking, distancing to at $>6$ feet when space allowed (typically 3-6 feet during 5 day a week school), cohorting same students together, frequent surface cleaning, and hand-washing. Additionally, all CPS students were screened at building entry each morning for symptoms. Each school had a dedicated isolation room to immediately isolate any person with COVID-19-like symptoms. Each school had an assigned a full-time CHD school nurse. All teachers and staff were required to undergo COVID-19 testing twice, once before school began and once after students arrived in the classroom in the fall. The processes for teacher and staff screening testing were separate than the in-school symptomatic student testing described in this report.

\section{Documenting Potential Transmission}

Daily partner meetings were utilized to investigate in-school transmission in a structured way. We trained and deployed CHD nurses to assist CHD epidemiologists in the investigation of close contacts and determine likelihood of suspected transmissions. A case was considered a probable/confirmed transmission if two individuals with close contact became ill (either in the classroom or in an extracurricular activity).

\section{Results}

Cincinnati Public Schools held a total of 9 weeks of full in-person learning, and 13 hybrid in-person learning weeks (6 weeks in the fall and 7 weeks in the winter). On a typical hybrid day there were $~ 13,000$ students in CPS buildings. On a typical full in person day there were $~ 25,000$ students in CPS buildings, with the remainder of students opting for on-line learning.

\section{Outcomes}

The rate of COVID-19 cases among in-person students, teachers, and staff are displayed in the Fig. 1. During hybrid and in-person learning periods, there were a total of 1936 ill cases in students and teacher/staff. Case numbers during the remote learning period are not included in this report as they were believed to be underreported as reporting from families became intermittent when children were not attending in-person school. School-related transmission events were rarely documented, with $3.2 \%$ of cases resulting in subsequent probable/confirmed incidents of transmission in CPS settings ( $n=61$ transmission events). Incidents primarily originated with positive students $(n=53)$ and 8 incidents of transmission originating with staff. Transmission incidents 


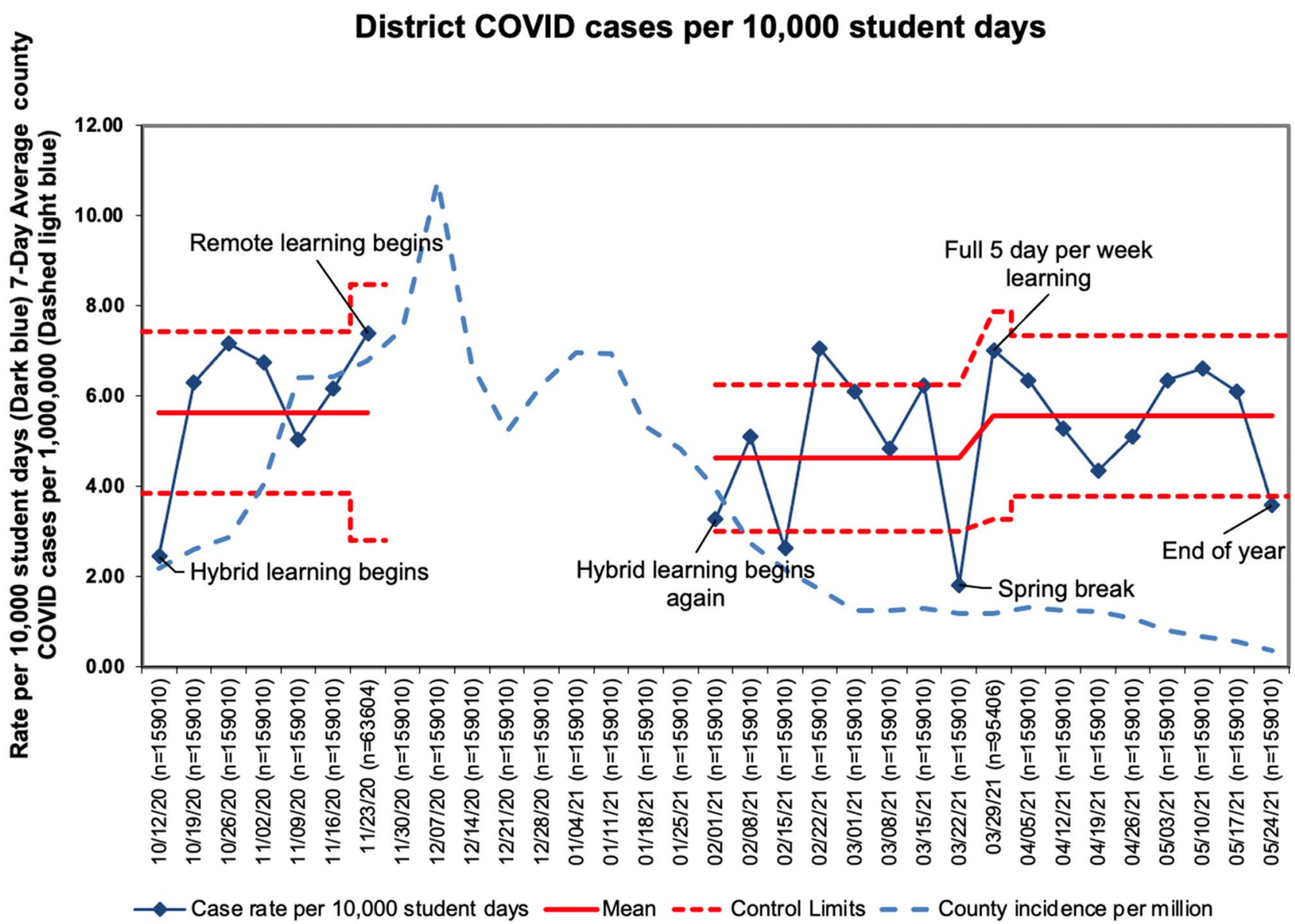

Fig.1 Number of cases in students and teachers per 10,000 learners during in-person learning. Solid blue line represents the number of cases in the schools per 10,000 in-person learners. The dashed blue line represents the number of cases in the county per 1,000,000 residents. Of note 10 community cases per $1,000,000$ equals 100 com- munity cases per 100,000. Each time period (first remote, second remote, and 5 day a week) has a new mean and control limits set as each period represented a fundamentally different system of learning (colour figure online)

Table 1 Performance on process measures

\begin{tabular}{|c|c|c|c|c|c|c|c|c|}
\hline & \multicolumn{4}{|c|}{ During Hybrid } & \multicolumn{4}{|c|}{ During 5 day a week school } \\
\hline & Number & $\begin{array}{l}\text { Number of } \\
\text { weeks }\end{array}$ & Number per week & $\%$ at goal $*$ & Number & $\begin{array}{l}\text { Number of } \\
\text { weeks }\end{array}$ & Number per week & $\%$ at goal $*$ \\
\hline $\begin{array}{l}\text { Number of children } \\
\text { in isolation room } \\
\text { per week }\end{array}$ & 2069 & 13 & 159.2 & 91 & 3688 & 9 & 409.8 & 90 \\
\hline $\begin{array}{l}\text { Number of Col- } \\
\text { laborative tests } \\
\text { performed per } \\
\text { week }\end{array}$ & 155 & 13 & 11.9 & 99 & 553 & 9 & 61.4 & 79 \\
\hline
\end{tabular}

* Goal for children in isolation room is to have them report to isolation room within 10 min of reporting feeling unwell. Goal for test results is that test result is reported to the school by 6AM the next day 
occurred predominantly in the classroom setting $(n=50)$, with the remaining 11 transmission incidents occurring in CPS extracurricular settings.

\section{Process measures}

The median time to isolation in the designated room for children reporting symptoms was consistently brief. Most children (>90\%) were isolated within $10 \mathrm{~min}$ of feeling unwell. (Table 1).

The number of children in the isolation room each day varied between hybrid and full 5 day teaching models, with 2069 children reporting to the isolation room during the 13 hybrid weeks (159.2 children per week) and 3688 children reporting to the isolation room during the 9 weeks of full in-person learning ( 409.8 children per week).

The number of COVID-19 tests performed by the school increased when students were in the classrooms 5 days a week compared with the hybrid period. During the hybrid period, there were 155 COVID tests performed over 13 weeks (average of 11.92 COVID tests performed per week). This number rose to a total of 553 COVID-19 tests performed over 9 weeks during the full 5 day a week period (61.4 tests per week).

The percentage of test results that were reported by 6AM the next day was very high during both learning models. $99 \%$ of all tests results were returned by $6 \mathrm{AM}$ during the hybrid period and $79 \%$ returned by $6 \mathrm{AM}$ during the 5 days a week period.

\section{Discussion}

Through a highly-intentional partnership between a large public school district, a city health department, and a freestanding children's hospital, we successfully implemented a robust set of COVID-19 protocols which included reliable, in-school access to COVID-19 PCR testing, next-day test result reporting and immediate quarantine of all exposed individuals. Testing in schools gave children access to a reliable, easily available testing location.

The use of QI tools including process map, and run charts allowed us to address complex processes and create consistent messages for teachers and staff. Reviewing the process maps with users prior to school beginning allowed them to visualize deficits. In times of uncertainty, process maps reassure school workers that plans do exist and encourage questions prior to the unfolding of actual events. The detailed, daily huddles to review of positive cases allowed for consideration of modifications to protocols. For example, one potential case of staff-to-staff transmission was reviewed, and job responsibilities were reconfigured to ensure more physical distancing in the future. The huddles allowed for daily learning and rapid mitigation for processes that might have failed. For significant challenges, questions and concerns were quickly escalated to senior leadership for communication across the district.

The collaborative faced several challenges in implementing processes. For example, we recognized the need for test result communication that was rapid and accessible, yet securely maintained confidentiality. Thus, we intentionally developed redundancy through both the health department and the school for positive case reporting using secure email and faxing systems. A second challenge was developing a tracking system for positive cases and close contacts that was both secure and accessible by critical team members in each organization. A cloud-based secure site was developed and modified as the team learned together. A third challenge included adequate staffing for testing and contact tracing. CPS hired enough nurses to staff a CHD nurse in every school building. Many of these nurses had previous experience as contact tracers early in the pandemic and were experienced with pandemic protocols. However, at times of the year the demand on all parts of the system was high.

Moving forward, this learning system approach can be adapted to focus on new shared outcomes. The proactive testing structure with close contact tracing prevented spread beyond only a few dozen probable transmissions in a large urban school district. This approach continues into the 2021-2022 year, maintaining a focus on minimizing in-school transmission and improving vaccine access for children. A multilayered COVID-19 mitigation approach with strong partnerships allows children to attend school while limiting COVID-19 transmission events, as evidenced by our experiences and others [7].

\section{Conclusion}

A collaborative learning system built among the public school system, public health department, and a local children's hospital, COVID-19 to implement school processes including fast, reliable COVID-19 testing and rapid results reporting which supported early identification and quarantine of close contacts. This multi-sector learning health system helped ensure in-school COVID-19 transmission was rare.

Supplementary Information The online version contains supplementary material available at https://doi.org/10.1007/s10900-022-01067-7.

Author Contributions All authors participated in the design of the collaboration, have provided critical revisions, and have approved the final manuscript submission. KAA wrote the initial draft. 
Funding This work was conducted without funding from an external organization.

Data Availability Not applicable.

Code Availability Not Applicable.

\section{Declarations}

Conflict of interest The authors declare no competing interests.

Ethics Approval Exempt.

Consent to Participate Not applicable.

Informed Consent Not applicable.

\section{References}

1. Children and COVID-19: State-level data report. Accessed February 14, 2022. http://www.aap.org/en/pages/ 2019-novel-coron avirus-covid-19-infections/child ren-and-covid-19-state-level-data-report/
2. Stein-Zamir, C., Abramson, N., Shoob, H., Libal, E., Bitan, M., Cardash, T., Cayam, R., \& Miskin, I. (2020). A large COVID-19 outbreak in a high school 10 days after schools' reopening, Israel, May 2020. Euro Surveill, 25(29), 2001352.

3. Yung, C. F., Kam, K., Nadua, K. D., et al. (2021). Novel Coronavirus 2019 Transmission Risk in Educational Settings. Clinical Infectious Diseases, 72(6), 1055-1058. https://doi.org/10.1093/ $\mathrm{cid} / \mathrm{ciaa} 794$

4. Achievement gap and coronavirus McKinsey. Accessed August 24, 2021. https://www.mckinsey.com/industries/public-andsocial-sector/our-insights/covid-19-and-student-learning-in-theunited-states-the-hurt-could-last-a-lifetime

5. COVID-19 Guidance for Safe Schools. Accessed August 24, 2021. http://www.aap.org/en/pages/2019-novel-coronavirus-covid-19infections/clinical-guidance/covid-19-planning-considerationsreturn-to-in-person-education-in-schools/

6. Guidance for COVID-19 Prevention in K-12 Schools CDC. Accessed August 24, 2021. https://www.cdc.gov/coronavirus/ 2019-ncov/community/schools-childcare/k-12-guidance.html

7. Zimmerman, K. O., Brookhart, M. A., Kalu, I. C., Boutzoukas, A. E., McGann, K. A., Smith, M. J., Maradiaga Panayotti, G. M., Armstrong, S. C., Weber, D. J., Moorthy, G. S., \& Benjamin, D. K. (2021). Community SARS-CoV-2 surge and withinschool transmission. Pediatrics. https://doi.org/10.1542/peds. 2021-052686

Publisher's Note Springer Nature remains neutral with regard to jurisdictional claims in published maps and institutional affiliations. 